\title{
Associations between the dietary patterns of pregnant Malaysian women and ethnicity, education, and early pregnancy waist circumference: A prospective cohort study
}

\author{
Heng Yaw Yong ${ }^{1}$, Zalilah Mohd Shariff ${ }^{15}$, Barakatun Nisak Mohd Yusof ${ }^{1}$, Zulida Rejali', Jacques Bindels ${ }^{3}$, Yvonne Yee Siang Tee \\ and Eline M. van der Beek ${ }^{3,5}$ \\ ${ }^{1}$ Department of Nutrition and Dietetics, Faculty of Medicine and Health Sciences, Universiti Putra Malaysia, 43400 Selangor, Malaysia \\ ${ }^{2}$ Department of Obstetrics and Gynaecology, Faculty of Medicine and Health Sciences, Universiti Putra Malaysia, 43400 Selangor, Malaysia \\ ${ }^{3}$ Danone Nutricia Research, 3584 Utrecht, The Netherlands \\ ${ }^{4}$ Danone Dumex (M) ShdBhd, Nilai Industrial Estate, 71800 Nilai, Negeri Sembilan, Malaysia \\ ${ }^{5}$ Department of Pediatrics, University Medical Centre Groningen, University of Groningen, 9700 RB Groningen, The Netherlands
}

BACKGROUND/OBJECTIVES: Little is known about the dietary patterns (DPs) of women during pregnancy. The present study aimed to identify the DPs of pregnant Malaysian women and their associations with socio-demographic, obstetric, and anthropometric characteristics.

SUBJECTS AND METHODS: This prospective cohort study included 737 participants enrolled in Seremban Cohort Study between 2013 and 2015. Food consumption was assessed using a validated 126-food item semi-quantitative food frequency questionnaire (SFFQ) at four time-points, namely, pre-pregnancy and at each trimester (first, second, and third). Principal component analysis (PCA) was used to identify DPs.

RESULTS: Three DPs were identified at each time point and designated DP 1-3 (pre-pregnancy), DP 4-6 (first trimester), DP 7-9 (second trimester) and DP 10-12 (third trimester). DP 1, 4, and 7 appeared to be more prudent diets, characterized by higher intakes of nuts, seeds \& legumes, green leafy vegetables, other vegetables, eggs, fruits, and milk \& dairy products. DP 2, 5, 8, and 11 had greater loadings of condiments \& spices, sugar, spreads \& creamer, though DP 2 had additional sweet foods, DP 5 and 8 had additional oils \& fats, and DP 11 had additional tea \& coffee, respectively. DP 3 and 6 were characterized by high protein (poultry, meat, processed, dairy, eggs, and fish), sugars (mainly as beverages and sweet foods), and energy (bread, cereal \& cereal products, rice, noodles \& pasta) intakes. DP 9 had additional fruits. However, DP 12 had greater loadings of energy foods (bread, cereal \& cereal products, rice, noodles \& pasta), sugars (mainly as beverages, and sweet foods), and good protein sources (eggs, nuts, seeds \& legumes). Malays were more likely to have lower adherence (LA) for DP 1 and 10 than non-Malays. DP 2, 8, and 11 were more prevalent among Malays than non-Malays. Women with a higher education were more likely to have LA for DP 10, and women with a greater waist circumference at first prenatal visit were more likely to show LA for DP 11.

CONCLUSIONS: DPs observed in the present study were substantially different from those reported in Western populations. Information concerning associations between ethnicity, waist circumference and education with specific DPs before and throughout pregnancy could facilitate efforts to promote healthy dietary behavior and the overall health and well-being of pregnant women.

Nutrition Research and Practice 2019;13(3):230-239; https://doi.org/10.4162/nrp.2019.13.3.230; pISSN 1976-1457 elSSN 2005-6168

Keywords: Dietary patterns, food frequency questionnaire, principal component analysis, pregnancy, Malaysian

\section{INTRODUCTION}

Diet significantly contributes to human health [1], and during pregnancy, dietary energy and nutrient requirements, specifically micronutrient requirements, are generally greater to support increased maternal metabolism and blood volume, red cell mass expansion, and to secure the delivery of nutrients to the fetus
[2]. Poor nutrition during pregnancy is indicative of greater short- and long-term health risks to both mother and child [3,4]. Potential health risks to women and their off-spring necessitate continuous monitoring of the nutritional statuses and dietary intakes of pregnant women. Moreover, because a healthy diet reduces the risk of short- and long-term adverse pregnancy outcomes [5], the identification of the optimal diets during

\footnotetext{
This study was conducted using data collected during the Seremban Cohort Study (SECOST) and was supported by a research grant from Danone Dumex (Malaysia) Shd. Bhd.

${ }^{\S}$ Corresponding Author: Zalilah Mohd Shariff, Tel. 603-89472472, Fax. 603-89426769, Email. zalilahms@upm.edu.my

Received: January 28, 2019, Revised: February 19, 2019, Accepted: May 8, 2019

This is an Open Access article distributed under the terms of the Creative Commons Attribution Non-Commercial License (http://creativecommons.org/licenses/by-nc/3.0/) which permits unrestricted non-commercial use, distribution, and reproduction in any medium, provided the original work is properly cited.
} 
pregnancy is an important public health issue.

Dietary intakes during pregnancy are commonly assessed using intakes of energy, macronutrients, micronutrients, and food groups [6,7] and maternal diet imbalance suggests poor nutritional status. In recent years, the focus of dietary studies has shifted from evaluations of single nutrients toward evaluations of dietary patterns, which describe combinations of commonly consumed foods [8]. This approach allows diets to be described holistically and the roles played by social and economic factors with respect to compliance with dietary patterns to be elucidated [9]. Dietary patterns may also help capture the complexities of diet, which are often inaccessible by nutrient analysis. The latter point is relevant because nutritional health outcomes are often the result of multiple synergies between nutrients and foods rather than the summed effects of individual components [10].

Previous studies have shown that nutrition prior to and during pregnancy may critically effect maternal health and subsequent fetal development [11-13]. A dietary pattern high in fruits and vegetables is associated with a reduced risk of preterm delivery [14-17], whereas dietary patterns characterized by high energy, saturated and trans fats, refined sugar, and sodium are associated with higher risks of preterm delivery [18], low birth weight [19], and small-for-gestational-age (SGA) infants [20]. Maternal diet not only affects fetal development but also infant risk of chronic disease in later life [21-23]. Mediterranean diets and diets high in fruits and vegetables have been found to protect allergies in children [24,25], while maternal diets high in white bread, red and processed meat, French fries, fried chicken, and vitamin $C$ rich drinks have been reported to increase the risks of childhood overweightedness and obesity significantly at 3 years of age [16].

Although several approaches have been used to identify DPs among pregnant women, the posterior approach derived from principal component analysis is the most commonly used [26-29]. However, most studies on the topic have been conducted in Western populations, and due to diet and lifestyle behavior differences, the dietary pattern exhibited by pregnant Malaysian women is certain to differ. In addition, the majority of nutritional studies focused on one of the phases of pregnancy, such as pre-pregnancy [30,31] or mid-to-late pregnancy [32,33], and as maternal diets appear to change during pregnancy. Furthermore, we should understand dietary changes during this period, as dietary changes during pregnancy appear to reflect women's efforts to balance physiological changes in an effort to secure a healthy outcome. However, such information is unavailable for pregnant Malaysian women. In addition, understanding the characteristics of pregnant women with different dietary patterns is important for devising appropriate strategies to promote healthy eating. Thus, the present study was undertaken to determine dietary patterns before and during pregnancy and to document the consistencies of and deviations from dietary patterns over time and the maternal factors associated with observed patterns.

\section{MATERIALS AND METHODS}

\section{Study design and location}

This study was performed using data from the Seremban Cohort Study (SECOST). The methodology of this study has been previously described in detail (http://dx.doi.org/10.1136/ bmjopen-2017-018321) [34]. In brief, SECOST is an on-going prospective cohort study in which eligible pregnant women in the first trimester $\left(<10^{\text {th }}\right.$ weeks of gestation) of pregnancy are recruited from three Maternal and Child Health $(\mathrm{MCH})$ clinics in Seremban District, Negeri Sembilan and then followed-up for more than two years postnatally. The primary objective of SECOST is to identify the determinants and pregnancy outcomes of maternal glycemia. In the present study, we included data from 737 pregnant women enrolled in SECOST. However, due to follow-up visit failures, data were only available for 737 women (pre-pregnancy), 629 women (first trimester), 515 women (second trimester) and 487 women (third trimester). No difference was evident between the socio-demographic or obstetric information of completers and non-completers.

The study protocol was approved by the Medical Research Ethics Committee (MREC), Universiti Putra Malaysia (UPM/FPSK/ 100-9/2-MJKEtika), and the Medical Research Ethics Committee (MREC), Ministry of Health Malaysia (KKM/NIHSEC/08/0804/ P12-613). Permission to conduct this study was also obtained from the Seremban District Health Office. All women provided written informed consent.

\section{Dietary assessment}

Dietary intake was assessed using a modified 126-food item semi-quantitative food frequency questionnaire (SFFQ), which represented foods consumed by Malaysians [35], to assess dietary intakes over the previous 30 days. However, this questionnaire does not provide information concerning the preparation of food, including whether food items are raw or cooked. The SFFQ has been shown to have good validity and reproducibility for documenting food intakes of pregnant Malaysian women [36]. The questionnaire, which addressed serving sizes and frequencies of consumption (average times per day, week, or month) of each food item, was completed by trained interviewers during face-to-face interviews. Amounts of consumed food items were then converted into servings (grams) per day for each food group [37].

Food items were categorized into 17 food types (Supplementary Table 2) on the basis of nutrient characteristics, culinary usage, and habitual intake, and information from previous Malaysian studies [36-38]. The food groups included in this study were: rice, noodles \& pasta; bread, cereal \& cereal products; poultry \& meat; processed meat; fish \& seafood; eggs; nuts, seeds \& legumes; milk \& dairy products; green leafy vegetables; other vegetables; fruits; tea $\&$ coffee; high energy beverages; sweet foods; sugar, spread \& creamer; condiments \& spices; and oils \& fats. The Malaysian Adult Nutrition Survey (MANS) showed that milk \& dairy product $(0.14$ servings per day) consumption by Malaysian adults was well below the recommended intake of 1-2 servings per day [37]. Similarly, we also found that pregnant women only consumed of 0.09 to 0.50 servings per day of milk \& dairy products. For this reason, we study did not differentiate between high-fat and low-fat milk and dairy products. Whole grain varieties are limited in Malaysia due to price and availability for purchasing. Red meats are 
included in "poultry \& meat", as both are considered to be similar protein sources. Furthermore, poultry consumption was much higher and more frequent than red meat consumption in our cohort, presumably because of the cost of red meat and food habits. Thus, types of meat were not separated into different categories. Palm oil is the most commonly used cooking oil in Malaysia, and thus, vegetable oils (oils/fats other than palm oil) were not separated from animal fats (ghee and butter), because the consumption of animal fats in our cohort was minimal.

Principal component analysis (PCA) was performed on 17 food groups to derive DPs. Factors were rotated by orthogonal transformation (varimax rotation) to maintain uncorrelated factors and for greater interpretability. Data suitability was assessed prior to PCA. Kaiser-Meyer-Olkin values were 0.68 (pre-pregnancy), $0.74\left(1^{\text {st }}\right.$ trimester $), 0.65$ ( $2^{\text {nd }}$ trimester $)$, and 0.66 ( $3^{\text {rd }}$ trimester), which exceeded the recommended value of 0.60 . The number of factors was determined using an eigen value cut-off of 1.5 [39]. As a rule of thumb, to assess the significance of factor loadings, a minimum factor loading of 0.30 is accepted [40]. Thus, food groups with a factor loading of $<0.3$ were eliminated from further analysis. Dietary patterns at each time point were designated DP 1-3 (pre-pregnancy), DP4-6 (first trimester), DP 7-9 (second trimester), and DP 10-12 (third trimester) (Table 2). The factor scores for each dietary pattern were calculated by summing the intakes of food items weighted by their factor loadings [41]. A high factor score (either positive or negative) indicated either high or low intake of that food group in dietary pattern. No negative factor score was observed for any DP in this study. Factor scores were then categorized into tertiles $\left(1^{\text {st }}\right.$ tertile $=$ low adherence $(L A), 2^{\text {nd }}$ tertile $=$ moderate adherence (MA), and $3^{\text {rd }}$ tertile = high adherence $(\mathrm{HA})$ ) to ease interpretations during subsequent analysis. Adherence was defined as the extent to which the subjects followed a particular DP.

Socio-demographic and obstetric characteristics of women

Socio-demographic variables included age, ethnicity, education, occupation, and monthly household income, and obstetrical variables included gestational weeks at first prenatal visit, parity, histories of gestational diabetes mellitus (GDM) and pre-eclampsia, family histories of diabetes mellitus and hypertension, and current pregnancy planning.

\section{Anthropometric measurements}

The waist circumference (WC) was measured at first prenatal visit (mean $9.67 \pm 2.55$ gestational weeks) using an SECA non-stretchable fiber measuring tape, and results were categorized as normal $(<80 \mathrm{~cm})$ or increased risk of metabolic complications $(\geq 80 \mathrm{~cm})$ [42]. Self-reported pre-pregnancy weights and measured heights were used to calculate pre-pregnancy body mass indices (BMI) $\left(\mathrm{kg} / \mathrm{m}^{2}\right)$ [pre-pregnancy weight $(\mathrm{kg}) /$ height $\left.(\mathrm{m})^{2}\right]$. These pre-pregnancy BMls were then classified as underweight $\left(<18.5 \mathrm{~kg} / \mathrm{m}^{2}\right)$, normal $(18.5-24.9$ $\left.\mathrm{kg} / \mathrm{m}^{2}\right)$, overweight $\left(25.0-29.9 \mathrm{~kg} / \mathrm{m}^{2}\right)$, or obese $\left(\geq 30.0 \mathrm{~kg} / \mathrm{m}^{2}\right)$ using the World Health Organization criteria [43].

\section{Statistical analysis}

The analysis was performed using IBM SPSS version 22 [44] Continuous and categorical variables are presented as descriptive statistics. All continuous variables were tested for normality using a histogram and the Shapiro-Wilk test. Univariate logistic regression was performed to assess the associations between DPs and sociodemographic, obstetric, and anthropometric measurements. Crude odds ratios were then compared with adjusted odds ratios. A variable was considered a confounder if its odds ratio changed by $\geq 10 \%$ after adjustment [45]. All variables with a $P$-value $<0.25$ by univariate analyses were entered into multivariate models as independent factors with age, family history of diabetes mellitus, family history of hypertension, medical history of GDM, medical history of pre-eclampsia, and parity as covariates [46]. Prior to the main analysis, data were screened and tested to ensure that the assumptions of statistical tests were met. Statistical significance was accepted for two-sided $P$-values of $<0.05$.

\section{RESULTS}

The characteristics of the 737 study subjects are summarized in Table 1. Mean age at study entry was $30.34 \pm 4.55$ years. Most

Table 1. Characteristics of the study subjects $(n=737)$

\begin{tabular}{|c|c|c|c|}
\hline \multirow{2}{*}{ Characteristics } & \multicolumn{2}{|c|}{ No. of participants } & \multirow{2}{*}{ Mean \pm SD } \\
\hline & $\mathrm{n}$ & (\%) & \\
\hline \multicolumn{4}{|l|}{ Socio-demographic information } \\
\hline Age at study entry (yrs) & & & $30.34 \pm 4.55$ \\
\hline$\leq 20$ & 9 & (1.2) & \\
\hline $21-30$ & 412 & $(55.9)$ & \\
\hline $31-40$ & 297 & (40.3) & \\
\hline$>40$ & 19 & (2.6) & \\
\hline \multicolumn{4}{|l|}{ Ethnicity } \\
\hline Malay & 658 & (89.3) & \\
\hline Chinese & 35 & (4.7) & \\
\hline Indian and others & 44 & $(6.0)$ & \\
\hline Education (yrs) & & & $13.08 \pm 2.43$ \\
\hline Secondary and lower & 329 & (44.6) & \\
\hline STPM/ Matric/ Diploma/ Certificate & 236 & (32.0) & \\
\hline Tertiary and above & 172 & (23.4) & \\
\hline
\end{tabular}


Table 1. continued

\begin{tabular}{|c|c|c|c|}
\hline \multirow{2}{*}{ Characteristics } & \multicolumn{2}{|c|}{ No. of participants } & \multirow{2}{*}{ Mean \pm SD } \\
\hline & $\mathrm{n}$ & $(\%)$ & \\
\hline \multicolumn{4}{|l|}{ Occupation } \\
\hline Unemployed & 223 & (30.3) & \\
\hline Employed & 514 & (69.7) & \\
\hline Monthly household income (MYR) ${ }^{1)}$ & & & $3,791.70 \pm 2,037.47$ \\
\hline Low $(<3,860)$ & 448 & $(60.8)$ & \\
\hline Middle $(3,860-8,319)$ & 268 & (36.4) & \\
\hline High $(\geq 8,320)$ & 21 & (2.8) & \\
\hline \multicolumn{4}{|l|}{ Obstetrical information } \\
\hline Gestational week at booking (weeks) & & & $9.67 \pm 2.55$ \\
\hline Parity & & & $1.23 \pm 1.28$ \\
\hline Nulliparous & 264 & (35.8) & \\
\hline Primiparous & 224 & (30.4) & \\
\hline Multiparous & 249 & (33.8) & \\
\hline \multicolumn{4}{|l|}{ Medical history } \\
\hline GDM & 60 & (8.1) & \\
\hline Pre-eclampsia & 20 & (2.7) & \\
\hline \multicolumn{4}{|l|}{ Family history } \\
\hline Diabetes mellitus & 199 & $(27.0)$ & \\
\hline Hypertension & 170 & (23.1) & \\
\hline \multicolumn{4}{|l|}{ Anthropometric measurements } \\
\hline Height $(m)$ & & & $1.56 \pm 0.06$ \\
\hline$<1.55$ & 270 & (36.6) & \\
\hline $1.55-1.58$ & 230 & (31.2) & \\
\hline$>1.58$ & 237 & $(32.2)$ & \\
\hline Waist circumference at first prenatal visit $(\mathrm{cm})$ & & & $78.69 \pm 10.52$ \\
\hline$<80$ & 439 & (59.6) & \\
\hline$\geq 80$ & 298 & (40.4) & \\
\hline Pre-pregnancy weight (kg) & & & $58.71 \pm 12.98$ \\
\hline Pre-pregnancy BMI $\left(\mathrm{kg} / \mathrm{m}^{2}\right)$ & & & $23.97 \pm 4.95$ \\
\hline Underweight $(<18.5)$ & 72 & (9.8) & \\
\hline Normal (18.5-24.9) & 399 & (54.1) & \\
\hline Overweight (25.0-29.9) & 171 & (23.2) & \\
\hline Obese $(\geq 30.0)$ & 95 & $(12.9)$ & \\
\hline
\end{tabular}

1) Economic Planning Unit, Prime Minister's Department, 2014. One USD was equivalent to 4.22 Malaysian Ringgit (MYR) at the time of the study. GDM: gestational diabetes mellitus.

Table 2. Factor loadings of dietary patterns at pre-pregnancy and during pregnancy

\begin{tabular}{|c|c|c|c|c|c|c|c|c|c|c|c|c|}
\hline \multirow{2}{*}{ Food groups } & \multicolumn{3}{|c|}{ Pre-pregnancy $(n=737)$} & \multicolumn{3}{|c|}{ First trimester $(n=629)$} & \multicolumn{3}{|c|}{ Second trimester $(n=515)$} & \multicolumn{3}{|c|}{ Third trimester $(n=487)$} \\
\hline & DP 1 & DP 2 & DP 3 & DP 4 & DP 5 & DP 6 & DP 7 & DP 8 & DP 9 & DP 10 & DP 11 & DP 12 \\
\hline Other vegetables & 0.73 & & & 0.81 & & & 0.76 & & & 0.65 & & \\
\hline Nuts, seeds \& legumes & 0.68 & & & 0.32 & & & 0.39 & & & & & 0.41 \\
\hline Green leafy vegetables & 0.57 & & & 0.43 & & & 0.80 & & & 0.72 & & \\
\hline Eggs & 0.57 & & & & & 0.59 & & & 0.54 & & & 0.52 \\
\hline Fruits & 0.41 & & & 0.43 & & & & & 0.56 & & & 0.65 \\
\hline Milk \& dairy products & 0.39 & & & & & 0.57 & & & 0.51 & & & 0.47 \\
\hline Condiments \& spices & & 0.97 & & & 0.96 & & & 0.96 & & & 0.97 & \\
\hline Sugar, spread \& creamer & & 0.97 & & & 0.96 & & & 0.96 & & & 0.97 & \\
\hline Sweet foods & & 0.31 & & & & 0.47 & & & 0.38 & & & 0.56 \\
\hline Rice, noodles \& pasta & & & 0.76 & & & & 0.40 & & & 0.55 & & \\
\hline Oils \& fats & & & 0.58 & & 0.33 & & & 0.31 & & & & \\
\hline High energy beverages & & & 0.53 & & & 0.38 & & & 0.54 & & & 0.51 \\
\hline Poultry \& meat & & & 0.51 & & & 0.35 & & & 0.38 & 0.44 & & \\
\hline Fish \& seafood & & & 0.49 & & & 0.51 & & & 0.61 & & & \\
\hline Bread, cereal \& cereal products & & & & & & 0.63 & & & 0.62 & & & 0.52 \\
\hline Processed meat & & & & & & 0.34 & & & 0.41 & & & \\
\hline Tea and coffee & & & & & & & & & & & 0.39 & \\
\hline Total variance & $13.54 \%$ & $13.11 \%$ & $11.90 \%$ & $11.30 \%$ & $12.92 \%$ & $14.39 \%$ & $10.66 \%$ & $12.56 \%$ & $15.40 \%$ & $10.84 \%$ & $13.34 \%$ & $13.52 \%$ \\
\hline
\end{tabular}

Dietary patterns contained food groups with absolute factor loadings of $>0.300$. 
Table 3. Multivariate associations between women's characteristics and dietary patterns

\begin{tabular}{|c|c|c|c|c|c|c|}
\hline \multirow{4}{*}{ Pre-pregnancy } & \multicolumn{6}{|c|}{ Dietary pattern $(\mathrm{DP})^{1)}$} \\
\hline & \multicolumn{2}{|c|}{ DP 1} & \multicolumn{2}{|c|}{ DP 2} & \multicolumn{2}{|c|}{ DP 3} \\
\hline & LA & $\mathrm{HA}$ & LA & $\mathrm{HA}$ & LA & $\mathrm{HA}$ \\
\hline & \multicolumn{6}{|c|}{ Adjusted OR $[95 \% \mathrm{Cl}]$} \\
\hline \multicolumn{7}{|l|}{ Ethnicity } \\
\hline Non-Malay & 1.00 & 1.00 & 1.00 & 1.00 & 1.00 & 1.00 \\
\hline Malay & $3.10[1.65-5.83]^{*}$ & $1.64[0.95-2.82]$ & $0.52[0.30-0.91]^{*}$ & $1.23[0.64-2.37]$ & $1.04[0.56-1.93]$ & $0.63[0.36-1.12]$ \\
\hline \multicolumn{7}{|l|}{ Education (yrs) } \\
\hline Secondary and lower & 1.00 & 1.00 & 1.00 & 1.00 & 1.00 & 1.00 \\
\hline Tertiary and above" & $0.85[0.59-1.22]$ & $1.10[0.76-1.58]$ & $0.83[0.58-1.20]$ & $0.73[0.50-1.05]$ & $1.06[0.73-1.54]$ & $0.78[0.54-1.13]$ \\
\hline \multicolumn{7}{|c|}{ Monthly household income (RM) } \\
\hline Low & 1.00 & 1.00 & 1.00 & 1.00 & 1.00 & 1.00 \\
\hline Middle to high & $1.16[0.80-1.67]$ & $1.31[0.91-1.90]$ & $1.21[0.84-1.75]$ & $1.29[0.90-1.87]$ & $0.98[0.68-1.43]$ & $0.83[0.57-1.20]$ \\
\hline \multicolumn{7}{|l|}{ WC at first prenatal visit $(\mathrm{cm})$} \\
\hline$<80$ & 1.00 & 1.00 & 1.00 & 1.00 & 1.00 & 1.00 \\
\hline$\geq 80$ & $0.95[0.65-1.38]$ & 1.14 [0.79-1.66] & $1.13[0.78-1.64]$ & $0.86[0.59-1.26]$ & $0.98[0.67-1.42]$ & $0.81[0.56-1.18]$ \\
\hline \multicolumn{7}{|l|}{ Pre-pregnancy BMI $\left(\mathrm{kg} / \mathrm{m}^{2}\right)$} \\
\hline Underweight/normal & 1.00 & 1.00 & 1.00 & 1.00 & 1.00 & 1.00 \\
\hline Overweight/obese & $1.08[0.74-1.58]$ & $0.99[0.68-1.45]$ & $1.08[0.74-1.57]$ & $0.81[0.55-1.19]$ & $0.89[0.61-1.31]$ & $0.90[0.62-1.32]$ \\
\hline \multirow{2}{*}{ First trimester } & \multicolumn{2}{|c|}{ DP 4} & \multicolumn{2}{|c|}{ DP 5} & \multicolumn{2}{|c|}{ DP 6} \\
\hline & LA & $\mathrm{HA}$ & LA & $\mathrm{HA}$ & LA & $\mathrm{HA}$ \\
\hline \multicolumn{7}{|l|}{ Ethnicity } \\
\hline Non-Malay & 1.00 & 1.00 & 1.00 & 1.00 & 1.00 & 1.00 \\
\hline Malay & $0.69[0.36-1.32]$ & 0.76 [0.39-1.49] & $0.83[0.45-1.54]$ & $1.11[0.57-2.17]$ & $1.01[0.53-1.94]$ & $0.86[0.45-1.64]$ \\
\hline \multicolumn{7}{|l|}{ Education (yrs) } \\
\hline Secondary and lower & 1.00 & 1.00 & 1.00 & 1.00 & 1.00 & 1.00 \\
\hline Tertiary and above & $0.79[0.53-1.17]$ & $1.06[0.71-1.58]$ & $1.32[0.89-1.96]$ & 1.14 [077-1.69] & $0.82[0.55-1.22]$ & $1.06[0.71-1.58]$ \\
\hline \multicolumn{7}{|c|}{ Monthly household income (RM) } \\
\hline Low & 1.00 & 1.00 & 1.00 & 1.00 & 1.00 & 1.00 \\
\hline Middle to high & $0.84[0.57-1.25]$ & $0.95[0.64-1.42]$ & $1.43[0.96-2.13]$ & $1.15[0.77-1.72]$ & $0.79[0.53-1.18]$ & $0.94[0.63-1.40]$ \\
\hline \multicolumn{7}{|c|}{ WC at first prenatal visit $(\mathrm{cm})$} \\
\hline$<80$ & 1.00 & 1.00 & 1.00 & 1.00 & 1.00 & 1.00 \\
\hline$\geq 80$ & $1.06[0.70-1.59$ & $1.11[0.74-1.68]$ & $1.20[0.80-1.79]$ & $0.87[0.57-1.31]$ & $1.33[0.88-2.00]$ & $1.10[0.73-1.66]$ \\
\hline Pre-pregnancy BMI $\left(\mathrm{kg} / \mathrm{m}^{2}\right)$ & & & & & & \\
\hline Underweight/normal & 1.00 & 1.00 & 1.00 & 1.00 & 1.00 & 1.00 \\
\hline Overweight/obese & $1.06[0.70-1.61]$ & $1.15[0.76-1.74]$ & 1.32 [0.88-1.99] & $0.96[0.63-1.46]$ & $1.37[0.91-2.07]$ & $0.94[0.61-1.42]$ \\
\hline Serond trimecter & & & & & & \\
\hline 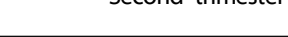 & LA & $\mathrm{HA}$ & LA & $\mathrm{HA}$ & LA & $\mathrm{HA}$ \\
\hline Ethnicity & & & & & & \\
\hline Non-Malay & 1.00 & 1.00 & 1.00 & 1.00 & 1.00 & 1.00 \\
\hline Malay & $1.76[0.83-3.72]$ & $0.99[0.52-1.91]$ & $0.56[0.29-1.06]$ & $2.36[1.01-5.63]^{*}$ & $1.30[0.64-2.62]$ & $1.03[0.53-2.02]$ \\
\hline Education (yrs) & & & & & & \\
\hline Secondary and lower & 1.00 & 1.00 & 1.00 & 1.00 & 1.00 & 1.00 \\
\hline Tertiary and above" & $1.23[0.79-1.91]$ & $1.16[0.75-1.81]$ & $0.80[0.52-1.25]$ & $1.03[0.66-1.59]$ & $0.98[0.63-1.52]$ & $0.93[0.60-1.44]$ \\
\hline Monthly household income & & & & & & \\
\hline Low & 1.00 & 1.00 & 1.00 & 1.00 & 1.00 & 1.00 \\
\hline Middle to high & $1.28[0.81-2.02]$ & 1.17 [0.75-1.84] & $1.44[0.92-2.26]$ & $1.16[0.74-1.83]$ & $0.93[0.60-1.45]$ & $0.92[0.59-1.44]$ \\
\hline WC at first prenatal visit ( & & & & & & \\
\hline$<80$ & 1.00 & 1.00 & 1.00 & 1.00 & 1.00 & 1.00 \\
\hline$\geq 80$ & $1.34[0.85-2.11]$ & $0.84[0.53-1.34]$ & $1.46[0.93-2.32]$ & $1.28[0.81-2.02]$ & $1.37[0.87-2.16]$ & $1.23[0.78-1.95]$ \\
\hline Pre-pregnancy BMI $\left(\mathrm{kg} / \mathrm{m}^{2}\right)$ & & & & & & \\
\hline Underweight/normal & 1.00 & 1.00 & 1.00 & 1.00 & 1.00 & 1.00 \\
\hline Overweight/obese & 1.09 [0.69-1.72] & $0.84[0.53-1.34]$ & $1.15[0.72-1.82]$ & $0.97[0.61-1.54]$ & $1.12[0.71-1.76]$ & $0.99[0.63-1.57]$ \\
\hline
\end{tabular}


Table 3. continued

\begin{tabular}{|c|c|c|c|c|c|c|}
\hline \multirow{2}{*}{ Third trimester } & \multicolumn{2}{|c|}{ DP 10} & \multicolumn{2}{|c|}{ DP 11} & \multicolumn{2}{|c|}{ DP 12} \\
\hline & LA & $\mathrm{HA}$ & LA & $\mathrm{HA}$ & LA & $\mathrm{HA}$ \\
\hline \multicolumn{7}{|l|}{ Ethnicity } \\
\hline Non-Malay & 1.00 & 1.00 & 1.00 & 1.00 & 1.00 & 1.00 \\
\hline Malay & $2.22[1.01-4.92]^{*}$ & $0.90[0.47-1.72]$ & $0.68[0.36-1.29]$ & $2.60[1.10-6.16]^{*}$ & $1.55[0.74-3.22]$ & $1.03[0.53-2.02]$ \\
\hline \multicolumn{7}{|l|}{ Education (yrs) } \\
\hline Secondary and lower & 1.00 & 1.00 & 1.00 & 1.00 & 1.00 & 1.00 \\
\hline Tertiary and above " & $1.77[1.13-2.78]^{*}$ & $1.54[0.98-2.41]$ & $0.83[0.53-1.29]$ & $1.04[0.66-1.62]$ & $0.66[0.42-1.04]$ & $1.40[0.98-2.20]$ \\
\hline \multicolumn{7}{|c|}{ Monthly household income (RM) } \\
\hline Low & 1.00 & 1.00 & 1.00 & 1.00 & 1.00 & 1.00 \\
\hline Middle to high & $1.71[0.99-2.73]$ & $1.25[0.78-1.99]$ & $1.50[0.94-2.38]$ & $1.13[0.71-1.81]$ & $0.72[0.45-1.15]$ & 1.11 [0.70-1.76] \\
\hline \multicolumn{7}{|c|}{ WC at first prenatal visit $(\mathrm{cm})$} \\
\hline$<80$ & 1.00 & 1.00 & 1.00 & 1.00 & 1.00 & 1.00 \\
\hline$\geq 80$ & $1.57[0.99-2.50]$ & $0.96[0.60-1.54]$ & $1.63[1.03-2.60]^{*}$ & $1.04[0.65-1.67]$ & $1.31[0.82-2.09]$ & 1.22 [0.76-1.95] \\
\hline \multicolumn{7}{|l|}{ Pre-pregnancy BMI $\left(\mathrm{kg} / \mathrm{m}^{2}\right)$} \\
\hline Underweight/normal & 1.00 & 1.00 & 1.00 & 1.00 & 1.00 & 1.00 \\
\hline Overweight/obese & $0.83[0.52-1.32]$ & $0.82[0.51-1.31]$ & 1.04 [0.65-1.67] & $0.80[0.50-1.29]$ & $0.98[0.61-1.57]$ & $0.81[0.50-1.31]$ \\
\hline
\end{tabular}

women were Malays (89.3\%), nulliparous (35.8\%), had completed secondary education (44.6\%), were employed (69.7\%), and were from low-income households (60.8\%). A small percentage of women reported having GDM (8.1\%) or pre-eclampsia $(2.7 \%)$ during a previous pregnancy. More than one-fifth had a family history of diabetes mellitus (DM) (27.0\%) or hypertension (23.1\%). About $40.4 \%$ had an early waist circumference measurement of $\geq 80 \mathrm{~cm}$, and $73.9 \%$ were overweight $(33.6 \%)$ or obese $(40.3 \%)$. More than half $(54.1 \%)$ reported a normal body mass index (BMI 18.50-24.99 kg/m²) before pregnancy.

The factor loadings of DPs before and during pregnancy (first, second, and third trimester) are provided in Table 2. Three DPs were identified at each time point: DP 1-3 for pre-pregnancy, DP 4-6 for the first trimester, DP 7-9 for the second trimester, and DP 10-12 for the third trimester. Pre-pregnancy, DP 1 was the most dominant food pattern and accounted for $13.5 \%$ of total dietary intake variation. During pregnancy, DP 6, DP 9 and DP 12 explained the largest percentage of total variance, with total variances of $14.4 \%, 15.4 \%$ and $13.5 \%$ for first, second, and third trimesters, respectively. DP 1 and DP 4 appeared to be a more prudent diet and were characterized by higher intakes of other vegetables, nuts, seeds \& legumes, green leafy vegetables, eggs, fruits, and milk \& dairy products. DP 7 in the second trimester became more plant- and staple food-based. However, in the third trimester, DP 10 was characterized by additional food sources choice rich in protein, such as poultry \& meat; nonetheless, this pattern was still considered healthy based on overall vegetable and fruit intakes. DP 2, 5, 8, and 11 had greater loadings of condiments \& spices, sugar, spread \& creamer, though DP 2 had additional sweet foods, DP 5 and DP 8 had additional oils \& fats, and DP 11 had additional tea \& coffee. DP 3 and DP 6 were characterized by high protein (poultry, meat, processed, dairy, eggs, and fish), sugars (mainly as beverages and sweet foods), and energy (bread, cereal \& cereal products, rice, noodles \& pasta). A similar pattern (DP 9) was found in the second trimester with additional fruits. However, in the third trimester, DP 12 had greater loadings of energy (bread, cereal \& cereal products, rice, noodles \& pasta), sugars (mainly as beverages, and sweet foods), and good protein sources (eggs and nuts, seeds \& legumes). Sensitivity analyses were performed on 487 pregnant women with complete data, and findings concurred with main findings (Supplementary Table 1).

Table 3 shows the associations between women's characteristics and DPs. Pre-pregnancy, women were more likely to have low adherence (LA) for DP 1, but less likely to have LA for DP 2. No significant association was found between maternal characteristics and DP during the first trimester, but during the second trimester, were more likely to have high adherence (HA) for DP8, and in the third trimester, were more likely to have LA for DP 10 and HA for DP11. Whereas, women with a higher waist circumference (WC) at first prenatal visit were more likely to have LA for DP 11. In addition, women with higher education were more likely to have LA for DP10.

\section{DISCUSSION}

The present study identified three distinct DPs in Malaysian pregnant women with specific adherence patterns over time and their dependence on maternal characteristics. Pre-pregnancy DP 1 was dominated by plant-based foods, eggs, milk and dairy products, and most resembled the DP commonly identified as "Prudent/Healthy" (vegetables, fruits, fish, legumes, whole grains, and dairy products) reported in Western studies [13,33,47-49]. 
DP 4, DP 7 and DP 10 during pregnancy remained somewhat plant-based with the addition of an additional staple-food in the second trimester and protein (poultry \& meat) in the third trimester. In a Korea study conducted on a general adult population, a healthy dietary pattern was characterized by high intakes of vegetables, fruits, whole grains, legumes, and seafood and low intakes of sweetened foods, refined grains, and processed meat [50]. However, during pregnancy, a healthy diet should be characterized as a balanced diet that includes a combination of all food groups to ensure that increased nutritional needs are met during pregnancy [51]. Thus, although rice, noodles \& pasta, and poultry \& meat were included in DP 7 and DP 10, these patterns were still considered as healthy. A similar pattern was reported in studies conducted in China ('vegetarian/vegetable pattern') and in the Growing Up in Singapore Towards Healthy Outcomes (GUSTO) study ('vegetable, fruit and white rice'). The patterns reported by these studies were characterized by high loadings of plant-based foods such as root vegetables, beans, leafy vegetables, fruits, and legumes $[17,32,33]$. Dietary patterns weighted toward plant-based foods (especially fruits), vegetables, whole grains, and lean meat throughout pregnancy have been shown to benefit both maternal and perinatal health $[2,13,33]$. He et al. [33] concluded vegetables and fruits were important components of protective dietary patterns and that a 'vegetable dietary pattern' was significantly associated with a lower risk of GDM [33]. In addition, Chia et al. [17] reported this dietary pattern was significantly associated with preterm birth and larger birth size [17].

DP2 and DP3 during pre-pregnancy, DP 5 and DP 6 during first trimester, DP 8 and DP 9 during second trimester, and DP 11 and DP 12 in third trimester were less healthy DPs characterized by either high intakes of fats and energy or protein and sugar. DP 3 changed from pre-pregnancy to pregnancy (DP 6, DP 9, and DP 12) to account for the largest proportion of total variance. Plausibly, preference for these energy dense foods is due to increased appetite during pregnancy. De-Graft Aikins [52] stated that Ghanaian women reported increased appetite during pregnancy and reported a craving for starch and protein rich foods [52]. In addition, many pregnant women believe that the consumption of high protein foods, such as meat, cheese, and milk, is important for infant growth and development [53]. Based on current knowledge, excessively low protein intake during pregnancy is associated with negative effects in terms of weight and length at birth. However, excessively high protein could also negatively impact fetal development [54]. Although fish \& seafood and eggs contain lower fat levels than meats, the methods used to prepare them may substantially increase fat contents. In Malaysia, fish \& seafood and eggs are usually deep-fried, grilled with oil, or with added oil and/or fat (e.g. curry, fried chili, coconut milk-based gravy, or sauce) [37]. In other words, increased fish \& seafood and egg intake may be associated with energy and fat intake increases.

DP 2 (pre-pregnancy), DP 5 (first trimester), DP 8 (second trimester), and DP 11 (third trimester) were much more consistent than other DP over time. This pattern was characterized by high loadings of condiments \& spices (at all time points), sugar, spread \& creamer (all time points), and lower loadings of oils
\& fats $\left(1^{\text {st }}\right.$ and $2^{\text {nd }}$ trimester) and tea $\&$ coffee ( $3^{\text {rd }}$ trimester). These DPs appear to be unique to the Malay population and are markedly different from those reported in Western populations $[13,33,47,49]$. This pattern appears to be similar to the 'less-healthy' pattern reported in pregnant women in the Universiti Sains Malaysia (USM) Birth Cohort Study [55]. The Malaysian Adult Nutrition Survey (MANS) also showed the majority of Malaysians consume tea (47\%) and coffee (28\%) at least once a day and habitually add sugar (four teaspoons per day) and creamer (three teaspoons per day) to beverages [37]. The condiments and spices included in this pattern were soy sauce (salty soy sauce or sweet soy sauce), oyster sauce, chili paste, and chili sauces, all of which are frequently used during food preparation. This finding illustrates specific dietary pattern differences between Asian and Western populations.

Although the present study showed Malays were more likely to have low adherence (LA) for DP 1 before pregnancy and DP 10 during the $3^{\text {rd }}$ trimester, average daily intakes of fruits and vegetables based on 24-hour dietary recall fell much below recommended levels. This finding is similar to that of the MANS, which reported that about $75.5 \%$ of Malaysian women aged 18 to 59 years did not meet the dietary guideline for fruit and vegetable intakes ( 3 to 5 servings per day) [37]. We also found that women with at least a tertiary education were significantly more likely to have LA for DP 10, but only in the third trimester. Previous studies showed that education level importantly enhances nutritional knowledge [56,57], and thus, it is possible that these women increased their energy and protein intakes in the $3^{\text {rd }}$ trimester to support fetal growth and development. However, whether this observation indicates that as pregnancy progresses, women with higher education become more aware of the relationship between diet and fetal growth and subsequently adopt a more diverse dietary pattern, requires further investigation.

We also found significant ethnic differences in DP 2 (condiments, spices, sugar, spread and creamer) during pre-pregnancy, DP 8 during the second trimester, and DP 11 during the third trimester, which probably reflect differences in food culture among ethnic groups. Malays were less likely to have LA before pregnancy but tended toward higher adherence (HA) to DP 8 and DP 11 than non-Malays. The main characteristic of Malay cuisine is the generous use of condiments and spices in dishes (either animal or plant-based). In addition, tea and coffee are served daily for breakfast and snacks and sugar is usually added to beverages. These findings highlight the importance of understanding dietary patterns of specific ethnic groups when designing targeted health promotion interventions.

In the third trimester, women with a higher WC at first prenatal visit were found to be more likely to have LA for DP 11 (condiments, spices, sugar, spread and creamer). About $73.9 \%$ of women with a WC of $\geq 80 \mathrm{~cm}$ at first prenatal visit were overweight $(33.6 \%)$ or obese $(40.3 \%)$ at study entry. Within the Malaysian health care setting, it is plausible that overweight or obese women received diet counseling from a health professional during pregnancy to reduce amounts of sugary foods, oil, and fats in diet to prevent excessive gestational weight gain (GWG) and pregnancy complications, such as gestational diabetes mellitus (GDM). Furthermore, previous 
studies have shown taste preferences may differ according to weight status, in that those who are overweight or obese appear show greater preference for energy-dense foods $[58,59]$.

This study has several limitations that warrant consideration. First, the study cohort was not representative of all pregnant Malaysian women. Most of the women enrolled were Malays, had a secondary education and lower, and were from low- or middle-income households. As, dietary patterns can vary by sex, socioeconomic status, ethnicity, and culture, we suggest the results of the study be compared with those of other pregnant Malaysian women representing various ethnic and socioeconomic groups. Second, the factor analysis and statistical methods used to define dietary patterns, for example, the consolidation of food items into food groups, the number of food groups included, and the number of factors extracted, were subjective. Sensitivity analysis was performed to assess robustness of results. Furthermore, all statistical analyses were checked for violation of the assumptions underlying those analyses (e.g., factor analysis) in order to minimize the potential bias. Third, use of the SFFQ for dietary assessments introduced the possibilities of recall bias and misreporting. However, the use of well-trained interviewers for data collection probably reduced these errors. Fourth, although the borderline significances of our findings caution that interpretations be approached carefully. Fifth we did not assess food preferences, food taboos, beliefs, or practices during pregnancy, or the social contexts of meal consumption. As these are important determinants of dietary intake, such information could explain adherence to eating patterns during pregnancy. Nevertheless, despite these limitations, we believe our findings provide important insights of the DPs of Malaysian women from pre-pregnancy and throughout pregnancy.

In conclusion, the DPs study differed substantially from those reported in Western populations. Furthermore, the different DPs observed before and during pregnancy, reflect clear changes in eating and drinking habits, although some healthy dietary adaptations did not last until the end of pregnancy. In addition, ethnicity, education, and WC in early pregnancy were significantly associated with specific DPs. These findings indicate the importance of understanding DPs when designing interventions aimed at promoting healthy diets for pregnant women. Such interventions should also consider the factors associated with the dietary intakes of pregnant women so that interventions can be tailored to meet the specific needs of women prior to and during pregnancy. Further studies are needed to determine the associations between DPs and pregnancy outcomes, child development, and later health.

\section{ACKNOWLEDGEMENTS}

We are extremely grateful to all women that participated in this study and to the medical officers and nurses in health clinics for their assistance during data collection.

\section{CONFLICTS OF INTERESTS}

The funder of this study was not involved in the study design or in any other aspect of the study. Jacques Bindels and Eline van der Beek are employees of Danone Nutricia Research and Yvonne Yee Siang Tee is an employee of Danone Dumex Malaysia. The authors declare that they have no proprietary, commercial, or financial interests that could be construed to have inappropriately influenced this study.

\section{ORCID}

Heng Yaw Yong: https://orcid.org/0000-0002-0454-641X

Zalilah Mohd Shariff: https://orcid.org/0000-0002-5347-4627

Barakatun Nisak Mohd Yusof: https://orcid.org/0000-0003-0403 $-5895$

Zulida Rejali: https://orcid.org/0000-0002-1386-5527

Jacques Bindels: https://orcid.org/0000-0001-7937-7107

Yvonne Yee Siang Tee: https://orcid.org/0000-0001-7129-5764

Eline M. van der Beek: https://orcid.org/0000-0002-7923-3653

\section{REFERENCES}

1. Nordström K, Coff C, Jönsson H, Nordenfelt L, Görman U. Food and health: individual, cultural, or scientific matters? Genes Nutr 2013;8:357-63.

2. Grieger JA, Clifton VL. A review of the impact of dietary intakes in human pregnancy on infant birthweight. Nutrients 2014;7:153-78.

3. Branca F, Piwoz E, Schultink W, Sullivan LM. Nutrition and health in women, children, and adolescent girls. BMJ 2015;351:h4173.

4. Kattula D, Sarkar R, Sivarathinaswamy P, Velusamy V, Venugopal S, Naumova EN, Muliyil J, Ward H, Kang G. The first 1000 days of life: prenatal and postnatal risk factors for morbidity and growth in a birth cohort in southern India. BMJ Open 2014;4:e005404.

5. Silva-Zolezzi I, Samuel TM, Spieldenner J. Maternal nutrition: opportunities in the prevention of gestational diabetes. Nutr Rev 2017;75:32-50.

6. Michels KB, Schulze MB. Can dietary patterns help us detect diet-disease associations? Nutr Res Rev 2005;18:241-8.

7. Liu FL, Zhang YM, Parés GV, Reidy KC, Zhao WZ, Zhao A, Chen C, Ning CY, Zheng YD, Wang PY. Nutrient intakes of pregnant women and their associated factors in eight cities of china: a cross-sectional study. Chin Med J (Engl) 2015;128:1778-86.

8. Hu FB. Dietary pattern analysis: a new direction in nutritional epidemiology. Curr Opin Lipidol 2002;13:3-9.

9. Dekker LH, Nicolaou M, van Dam RM, de Vries JH, de Boer EJ, Brants HA, Beukers MH, Snijder MB, Stronks K. Socio-economic status and ethnicity are independently associated with dietary patterns: the HELIUS-dietary patterns study. Food Nutr Res 2015;59:26317.

10. Cespedes EM, Hu FB. Dietary patterns: from nutritional epidemiologic analysis to national guidelines. Am J Clin Nutr 2015;101:899-900.

11. de Seymour J, Chia A, Colega M, Jones B, McKenzie E, Shirong C, Godfrey K, Kwek K, Saw SM, Conlon C, Chong YS, Baker P, Chong MF. Maternal dietary patterns and gestational diabetes mellitus in a multi-ethnic Asian cohort: the GUSTO study. Nutrients 2016;8: E574.

12. Chen $X$, Zhao D, Mao X, Xia Y, Baker PN, Zhang H. Maternal dietary patterns and pregnancy outcome. Nutrients 2016;8:E351.

13. Tryggvadottir EA, Medek H, Birgisdottir BE, Geirsson RT, Gunnarsdottir I. Association between healthy maternal dietary pattern and risk for gestational diabetes mellitus. Eur J Clin Nutr 2016;70:237-42. 
14. Lu MS, He JR, Chen Q, Lu J, Wei X, Zhou Q, Chan F, Zhang L, Chen N, Qiu L, Yuan M, Cheng KK, Xia H, Qiu X; Born in Guangzhou Cohort Study Group. Maternal dietary patterns during pregnancy and preterm delivery: a large prospective cohort study in China. Nutr J 2018;17:71.

15. Englund-Ögge L, Brantsæter AL, Sengpiel V, Haugen M, Birgisdottir BE, Myhre R, Meltzer HM, Jacobsson B. Maternal dietary patterns and preterm delivery: results from large prospective cohort study. BMJ 2014;348:g1446.

16. Martin CL, Siega-Riz AM, Sotres-Alvarez D, Robinson WR, Daniels JL, Perrin EM, Stuebe AM. Maternal dietary patterns during pregnancy are associated with child growth in the first 3 years of life. J Nutr 2016;146:2281-8.

17. Chia AR, de Seymour JV, Colega M, Chen LW, Chan YH, Aris IM, Tint MT, Quah PL, Godfrey KM, Yap F, Saw SM, Baker PN, Chong YS, van Dam RM, Lee YS, Chong MF. A vegetable, fruit, and white rice dietary pattern during pregnancy is associated with a lower risk of preterm birth and larger birth size in a multiethnic Asian cohort: the growing up in Singapore towards healthy outcomes (GUSTO) cohort study. Am J Clin Nutr 2016;104:1416-23.

18. Grieger JA, Grzeskowiak LE, Clifton VL. Preconception dietary patterns in human pregnancies are associated with preterm delivery. J Nutr 2014;144:1075-80.

19. Hajianfar H, Esmaillzadeh A, Feizi A, Shahshahan Z, Azadbakht L. Major maternal dietary patterns during early pregnancy and their association with neonatal anthropometric measurement. BioMed Res Int 2018;2018:4692193.

20. Okubo H, Miyake Y, Sasaki S, Tanaka K, Murakami K, Hirota Y; Osaka Maternal and Child Health Study Group, Kanzaki H, Kitada M, Horikoshi Y, Ishiko O, Nakai Y, Nishio J, Yamamasu S, Yasuda J, Kawai S, Yanagihara K, Wakuda K, Kawashima T, Narimoto K, Iwasa $\mathrm{Y}$, Orino K, Tsunetoh I, Yoshida J, lito J, Kaneko T, Kamiya T, Kuribayashi $\mathrm{H}$, Taniguchi T, Takemura $\mathrm{H}$, Morimoto $\mathrm{Y}$, Matsunaga I, Oda $\mathrm{H}$, Ohya $\mathrm{Y}$. Maternal dietary patterns in pregnancy and fetal growth in Japan: the Osaka maternal and child health study. $\mathrm{Br}$ J Nutr 2012;107:1526-33.

21. Murrin C, Shrivastava A, Kelleher CC; Lifeways Cross-generation Cohort Study Steering Group. Maternal macronutrient intake during pregnancy and 5 years postpartum and associations with child weight status aged five. Eur J Clin Nutr 2013;67:670-9.

22. Chen LW, Aris IM, Bernard JY, Tint MT, Colega M, Gluckman PD, Tan KH, Shek LP, Chong YS, Yap F, Godfrey KM, van Dam RM, Chong MF, Lee YS. Associations of maternal macronutrient intake during pregnancy with infant BMl peak characteristics and childhood BMI. Am J Clin Nutr 2017;105:705-13.

23. Wood-Bradley RJ, Henry SL, Vrselja A, Newman V, Armitage JA. Maternal dietary intake during pregnancy has longstanding consequences for the health of her offspring. Can J Physiol Pharmacol 2013;91:412-20.

24. Chatzi L, Torrent M, Romieu I, Garcia-Esteban R, Ferrer C, Vioque $J$, Kogevinas $M$, Sunyer J. Mediterranean diet in pregnancy is protective for wheeze and atopy in childhood. Thorax 2008;63: 507-13.

25. Willers SM, Devereux G, Craig LC, McNeill G, Wijga AH, Abou El-Magd W, Turner SW, Helms PJ, Seaton A. Maternal food consumption during pregnancy and asthma, respiratory and atopic symptoms in 5-year-old children. Thorax 2007;62:773-9.

26. Jarman $M$, Mathe N, Ramazani F, Pakseresht M, Robson PJ, Johnson
ST, Bell RC; APrON and ENRICH study teams. Dietary patterns prior to pregnancy and associations with pregnancy complications. Nutrients 2018;10:E914.

27. Zareei S, Homayounfar R, Naghizadeh MM, Ehrampoush E, Rahimi M. Dietary pattern in pregnancy and risk of gestational diabetes mellitus (GDM). Diabetes Metab Syndr 2018;12:399-404.

28. Rasmussen MA, Maslova E, Halldorsson TI, Olsen SF. Characterization of dietary patterns in the Danish national birth cohort in relation to preterm birth. PLoS One 2014;9:e93644.

29. Da Mota Santana J, Alves de Oliveira Queiroz V, Monteiro Brito S, Barbosa Dos Santos D, Marlucia Oliveira Assis A. Food consumption patterns during pregnancy: a longitudinal study in a region of the North East of Brazil. Nutr Hosp 2015;32:130-8.

30. Bao W, Bowers K, Tobias DK, Hu FB, Zhang C. Prepregnancy dietary protein intake, major dietary protein sources, and the risk of gestational diabetes mellitus: a prospective cohort study. Diabetes Care 2013;36:2001-8.

31. Schoenaker DA, Soedamah-Muthu SS, Callaway LK, Mishra GD. Pre-pregnancy dietary patterns and risk of gestational diabetes mellitus: results from an Australian population-based prospective cohort study. Diabetologia 2015;58:2726-35.

32. Yang J, Dang S, Cheng Y, Qiu H, Mi B, Jiang Y, Qu P, Zeng L, Wang Q, Li Q, Kang Y, Shen Y, Yan H. Dietary intakes and dietary patterns among pregnant women in Northwest China. Public Health Nutr 2017;20:282-93.

33. He JR, Yuan MY, Chen NN, Lu JH, Hu CY, Mai WB, Zhang RF, Pan YH, Qiu L, Wu YF, Xiao WQ, Liu Y, Xia HM, Qiu X. Maternal dietary patterns and gestational diabetes mellitus: a large prospective cohort study in China. Br J Nutr 2015;113:1292-300.

34. Yong HY, Mohd Shariff Z, Rejali Z, Mohd Yusof BN, Yasmin F, Palaniveloo L. Seremban cohort study (SECOST): a prospective study of determinants and pregnancy outcomes of maternal glycaemia in Malaysia. BMJ Open 2018;8:e018321.

35. Ministry of Health Malaysia. Malaysian Adult Nutrition Survey 2003. Putrajaya: Nutrition Section Family Health Development Division Ministry of Health Malaysia; 2007.

36. Loy SL, Marhazlina M, Nor AY, Hamid JJ. Development, validity and reproducibility of a food frequency questionnaire in pregnancy for the Universiti Sains Malaysia birth cohort study. Malays J Nutr 2011;17:1-18.

37. Norimah AK, Safiah M, Jamal K, Haslinda S, Zuhaida H, Rohida S, Fatimah S, Norazlin S, Poh BK, Kandiah M, Zalilah MS, Wan Manan WM, Fatimah S, Azmi MY. Food consumption patterns: findings from the Malaysian adult nutrition survey (MANS). Malays J Nutr 2008;14:25-39.

38. Nurliyana AR, Mohd Nasir MT, Zalilah MS, Rohani A. Dietary patterns and cognitive ability among 12 - to 13 year-old adolescents in Selangor, Malaysia. Public Health Nutr 2015;18:303-12.

39. Shadman Z, Poorsoltan N, Akhoundan M, Larijani B, Soleymanzadeh M, Akhgar Zhand C, Seyed Rohani ZA, Khoshniat Nikoo M. Ramadan major dietary patterns. Iran Red Crescent Med J 2014;16:e16801.

40. Marchioni DM, Latorre Mdo R, Eluf-Neto J, Wünsch-Filho V, Fisberg RM. Identification of dietary patterns using factor analysis in an epidemiological study in São Paulo. Sao Paulo Med J 2005;123: 124-7.

41. Hu FB, Rimm E, Smith-Warner SA, Feskanich D, Stampfer MJ, Ascherio A, Sampson L, Willett WC. Reproducibility and validity of dietary patterns assessed with a food-frequency questionnaire. Am 
J Clin Nutr 1999;69:243-9.

42. Nishida C, Ko GT, Kumanyika S. Body fat distribution and noncommunicable diseases in populations: overview of the 2008 WHO expert consultation on waist circumference and waist-hip ratio. Eur J Clin Nutr 2010;64:2-5.

43. Obesity: preventing and managing the global epidemic. Report of a WHO consultation. World Health Organ Tech Rep Ser 2000;894:i-xii, 1-253.

44. IBM Corp. Released. IBM SPSS Statistics for Windows, Version 23.0. Armonk (NY): IBM Corp.; 2015.

45. Greenland S. Modeling and variable selection in epidemiologic analysis. Am J Public Health 1989;79:340-9.

46. Hosmer DW, Lemeshow S. Applied logistic regression. In: Shewhart WA, Wilks SS, editors. Model-Building Strategies and Methods for Logistic Regression. Hoboken (NJ): John Wiley \& Sons, Inc.; 2000. p.91-142.

47. Bao W, Bowers K, Tobias DK, Olsen SF, Chavarro J, Vaag A, Kiely $M$, Zhang $C$. Prepregnancy low-carbohydrate dietary pattern and risk of gestational diabetes mellitus: a prospective cohort study. Am J Clin Nutr 2014;99:1378-84.

48. Loy SL, Marhazlina M, Jan JM. Association between maternal food group intake and birth size. Sains Malays 2013;42:1633-40.

49. Shin D, Lee KW, Song WO. Dietary patterns during pregnancy are associated with risk of gestational diabetes mellitus. Nutrients 2015;7:9369-82.

50. Baik I, Lee M, Jun NR, Lee JY, Shin C. A healthy dietary pattern consisting of a variety of food choices is inversely associated with the development of metabolic syndrome. Nutr Res Pract 2013;7: 233-41.

51. Cox JT, Phelan ST. Nutrition during pregnancy. Obstet Gynecol Clin
North Am 2008;35:369-83.

52. De-Graft Aikins A. Food beliefs and practices during pregnancy in Ghana: implications for maternal health interventions. Health Care Women Int 2014;35:954-72.

53. Yong HY, Mohd Shariff Z, Koo SJ, Binti Sa'ari NS. Pre-pregnancy body mass index, height and physical activity are associated with rate of gestational weight gain among Malaysian mothers. J Obstet Gynaecol Res 2016;42:1094-101.

54. Kramer MS, Kakuma R. Energy and protein intake in pregnancy. Cochrane Database Syst Rev 2003;CD000032.

55. Loy SL, Jan Mohamed HJ. Relative validity of dietary patterns during pregnancy assessed with a food frequency questionnaire. Int J Food Sci Nutr 2013;64:668-73.

56. Manaf ZA, Johari N, Mei LY, Yee NS, Yin CK, Teng LW. Nutritional status and nutritional knowledge of Malay pregnant women in selected private hospitals in Klang Valley. J Sains Kesihat Malays 2014;12:53-62.

57. Lim ZX, Wong JL, Lim PY. Knowledge of nutrition during pregnancy and associated factors among antenatal mothers. Int J Publ Health Clin Sci 2018;5:117-28.

58. Lanfer A, Knof K, Barba G, Veidebaum T, Papoutsou S, de Henauw S, Soós T, Moreno LA, Ahrens W, Lissner L. Taste preferences in association with dietary habits and weight status in European children: results from the IDEFICS study. Int J Obes (Lond) 2012;36:27-34.

59. Stelmach-Mardas $M$, Rodacki T, Dobrowolska-Iwanek J, Brzozowska A, Walkowiak J, Wojtanowska-Krosniak A, Zagrodzki P, Bechthold A, Mardas $M$, Boeing $\mathrm{H}$. Link between food energy density and body weight changes in obese adults. Nutrients 2016;8:229. 